\title{
Compound conditioning of separately pretrained conditioned stimuli evoking dissimilar conditioned responses
}

\author{
MARK J. BOURNE \\ Temple University, Philadelphia, Pennsylvania
}

(Todd R. Schachtman, Sponsor)

\begin{abstract}
The present experiment examined the effects of stimulus compounding of two appetitively and separately pretrained conditioned stimuli (CSs) in which each CS (light and tone) evoked a dissimilar conditioned response (CR). In Phase 1 , all rats received classical conditioning to the light and tone as separate stimulus elements. In Phase 2, one group received these two stimuli as a single reinforced compound stimulus and a control group continued to receive the stimuli reinforced separately. All subjects were then tested for responding to the single stimulus elements. In contrast to the predictions of current models of classical conditioning, it was found that compound conditioning of separately pretrained stimuli resulted in an increase in responding to the light, although there was a tendency toward a decrease in responding to the tone. Therefore, the rules governing the stimulus compounding of CSs with dissimilar CRs may be different from those governing CSs that evoke CRs with a similar response topography.
\end{abstract}

Kamin (1969) proposed that classical conditioning was a function of the extent to which the unconditioned stimulus (US) generated "surprise"' when presented immediately after the conditioned stimulus (CS). If the US is completely expected, as demonstrated by an asymptotic level of conditioned responding, the subject experiences no surprise and will not yield further conditioning. However, if a small conditioned response (CR) is observed relative to the amount of conditioning that the US can support, then a US will be surprising and a large amount of conditioning will take place to that particular CS. The notion of surprise and the development of conditioning theories based on expectancy and US effectiveness (e.g., Kamin, 1968; Rescorla \& Wagner, 1972; Terry \& Wagner, 1975; Wagner, Rudy, \& Whitlow, 1973) has led to novel predictions. For example, a loss of associative strength (conditioning) is predicted despite consistent reinforcement of a CS when that reinforcement is overexpected (Kremer, 1978; Rescorla \& Wagner, 1972; but see Schachtman, Kasprow, Chee, \& Miller, 1985). According to this view, when initially two different CSs are conditioned individually and then subsequently are reinforced as a compound $\mathrm{CS}$, the magnitude of the US is overpredicted, or overexpected, by the subjects. The Rescorla-Wagner model (Rescorla \& Wagner, 1972) claims that expectancies based

I wish to thank Lynn J. Hammond, to whom I owe a great debt for making this research possible. I also thank Joshua E. Blustein, for his critical reading of an earlier version of this manuscript and for statistical assistance, and Todd $\mathbf{R}$. Schachtman, for his encouragement and for reading a preliminary draft. Requests for reprints should be addressed to Mark J. Bourne, 210 McAlister Hall, University of MissouriColumbia, Columbia, MO 65211. on different stimuli quantitatively add together when those stimuli are presented in compound. Hence, it assumes that the subjects expect twice the magnitude of the US than actually occurs; this produces surprise. As a result of such trials, there will be a decrement in associative strength of the elements until the subjects' expectations match the amount of learning the US can actually support.

Rescorla (1970) demonstrated a loss of associative strength to a CS despite continuous reinforcement. Specifically, Rescorla used a conditioned barpress suppression procedure to individually condition a light and a tone CS to near asymptotic levels of conditioning. This procedure produced a similar CR (conditioned suppression) for each of the CSs. These CSs were then combined in a second phase to form a simultaneous compound CS that was followed by the same magnitude of shock as that received during the prior separate conditioning of each CS. After compound conditioning, the original elements were tested individually. The results obtained were consistent with the Rescorla and Wagner (1972) notion concerning the adjustment of expectancies when the US is overexpected on the compound CS conditioning trials. That is, there was a reduction in the fear elicited by each element as a result of compound conditioning relative to the fear elicited by each element before compound conditioning. This decrement in the CR was said to occur because the summation of the compound CS allowed the subjects to overpredict the magnitude of the US. However, while the Rescorla-Wagner model predicts the magnitude as well as the direction of these changes in associative strength as a result of compound training, some questions have been raised about this notion (Rescorla, 1989). Nonetheless, this prediction may depend upon the use of the same 
CR evoked by both CSs. The prediction has not been tested when different CRs occur to each CS as a result of the initial and separate training of cues. It is possible that the competition may follow different rules if that competition is no longer limited to a single CR dimension that is common to both independently trained CSs.

The specific nature of the US was previously thought to have been the primary, or perhaps even exclusive, factor in determining the form of the CR (Hilgard \& Marquis, 1940; Jenkins \& Moore, 1973; Rescorla \& Solomon, 1967). Thus, different CSs predicting a common US should yield the same kind of CR. Similarly, the behavioral effects of compounding two pretrained stimuli should be additive.

Holland and Rescorla (1975a, 1975b) reported that there were different levels of activity when subjects were presented with either a light or a tone CS. Holland (1977) tested the role of the CS modality on the topography of the conditioned response. He found that there were dissimilar forms of the CR to light and tone CSs (rearing and head-jerking, respectively). Consequently, the specific qualities of the CS appear to influence the form of the CR. To test the prediction of the Rescorla-Wagner model concerning overexpectation of the US magnitude and the loss of associative strength, the present study employed two pretrained CSs that each elicited a dissimilar CR (similar to the response topographies of Holland, 1977).

\section{METHOD}

\section{Subjects}

The subjects were 16 naive male Sprague-Dawley albino rats (Holtzman Co., Madison, WI). They were housed individually in stainless steel cages $(24 \times 20 \times 18 \mathrm{~cm})$ and exposed to $24 \mathrm{~h}$ of continuous light. They were maintained on a 23-h food-deprivation schedule, and were fed Purina Rat Chow for $\mathbf{1}$ h shortly after each experimental session. Water was available ad lib.

\section{Apparatus}

The experiment used four standard operant chambers (MSP-3000, BRS/LVE Inc., Laurel, MD). The chambers had two Plexiglas sidewalls, a Plexiglas ceiling, aluminum front and rear walls, and a shockgrid floor. The shock grids were approximately $0.5 \mathrm{~cm}$ in diameter and spaced $2 \mathrm{~cm}$ apart. The chamber measured $28 \times 20 \times 15 \mathrm{~cm}(1 \times w \times h)$. Mounted on the front wall were: a $24-\mathrm{V}$ dc intermittent light centered on the wall $10 \mathrm{~cm}$ above the grid floor; a Sonalert sound source (Model SC628) located $5.5 \mathrm{~cm}$ to the right of center of the wall and $10.5 \mathrm{~cm}$ above the grid floor; and a food magazine located $6 \mathrm{~cm}$ to the left of center of the wall and $3 \mathrm{~cm}$ above the grid floor. The food, $45-\mathrm{mg}$ pellets (P. J. Noyes Co.), was delivered into the magazine by means of a food cup that was connected to the food magazine. These stimulus events were preprogrammed on Telex tape and read by electromechanical programming equipment.

\section{Procedure}

Pretraining occurred on Day 1 of the study. During this session, the subjects were initially placed in the operant chambers for $15 \mathrm{~min}$ to acclimate them to the experimental context. All subjects received 30 trials of magazine training. The first half of the session (i.e., the first 15 reinforcers received) was conducted on a variable time 1-min (range = 0.5-1.5 min) schedule of reinforcement. The second half of the session (i.e., the last 15 reinforcers received) was conducted on a variable time 2-min (range $=0.5-3.0 \mathrm{~min}$ ) schedule of reinforcement.
On each of the next 3 days, a baseline response session was conducted. The subjects were given a total of 10 light- and 10 tone-stimulus presentations, separately and in random order. Each CS was presented for $10 \mathrm{sec}$. No food reinforcement was given. Such trials were separated by an intertrial interval of $5 \mathrm{~min}$ (range $=1.0-9.0 \mathrm{~min}$ ). During the $10-\mathrm{sec}$ stimulus presentation, the dominant form of the CR was recorded and categorized by a single observer seated approximately $2 \mathrm{~m}$ away. A checklist procedure, similar to that used by Bindra and Blond (1958), Shettleworth (1975), and Holland (1977), was used. In this procedure, the observer switches his/her visual inspection from chamber to chamber, starting with a different chamber on each trial. This ensured random observation over all CS presentations. Thus, one behavior per observation was recorded for each subject during the CS presentation, and was categorized by the form of the $\mathrm{CR}$. The behavioral categories employed in this study were the same as those used by Holland (1977) (see Appendix). The dominant behavior to each CS was recorded in these sessions.

Phase 1. The initial phase of conditioning was begun the day following the completion of the baseline sessions. Each subject was then randomly assigned to one of two groups $(n=8)$. Group LT received separate presentations of the light and the tone during the session. Group L/T received the same treatment as the experimental group during this phase of conditioning. During Phase 1, a total of 10 light and 10 tone exposures were presented in random order each session, for a total of six sessions. Each CS was $10 \mathrm{sec}$ in duration, and upon termination, was immediately followed by a food pellet. A variable intertrial interval with a mean length of $5 \mathrm{~min}$ (range $=1.0-9.0 \mathrm{~min}$ ) was used. The dominant behavior to each CS was recorded as noted previously.

Phase 2. During this phase of conditioning, Group LT, which previously had been conditioned to the light and the tone individually, received these elements as a reinforced compound CS for a total of 20 trials per session. That is, the light and the tone were presented simultaneously for $10 \mathrm{sec}$ and immediately followed by a food pellet. Group L/T continued to receive 10 light and 10 tone exposures, in random order, as separately presented stimuli. The groups were equated for the total number of reinforcements (i.e., 20 reinforcements for the compound CS for Group LT and 10 reinforcements for each of the light and the tone single elements for Group L/T). Since the single CSs were already assumed to be at associative asymptote as a result of the training received in Phase 1, the number of reinforcements per CS should not be a factor in determining the rate of conditioning. Moreover, according to the Rescorla-Wagner model, even if more opportunities for conditioning were possible in Group LT, this could be viewed as promoting more potential for producing a decrement in the CR due to overexpectation of the US. These trials were distributed as in Phase 1. The behavior was recorded as in Phase 1 .

Test. The test involved only one session and occurred the day after Phase 2 was completed. The test session served to measure any differences between the dominant forms of the CR for Groups LT and L/T. Secondly, and more importantly, this session also served to measure any changes in the magnitude and direction of associative strength as a consequence of compound conditioning. The test involved 1010 -sec nonreinforced presentations of the light and tone separately to all subjects. These exposures were separated by a 5 -min intertrial interval.

\section{RESULTS}

Conditioning of the light and the tone as a compound CS did result in changes in the dominant form of the CR. However, this treatment led to an increase in the probability of the dominant CR in the presence of the light and a reduction in the probability of the occurrence of the dominant CR in the presence of the tone. The assessment of the effects of compound conditioning of CS-dependent CRs involves comparing the magnitude of the CR with each CS before and after the compound conditioning phase, and then weighing these changes against further conditioning of the elements in the control group. The 

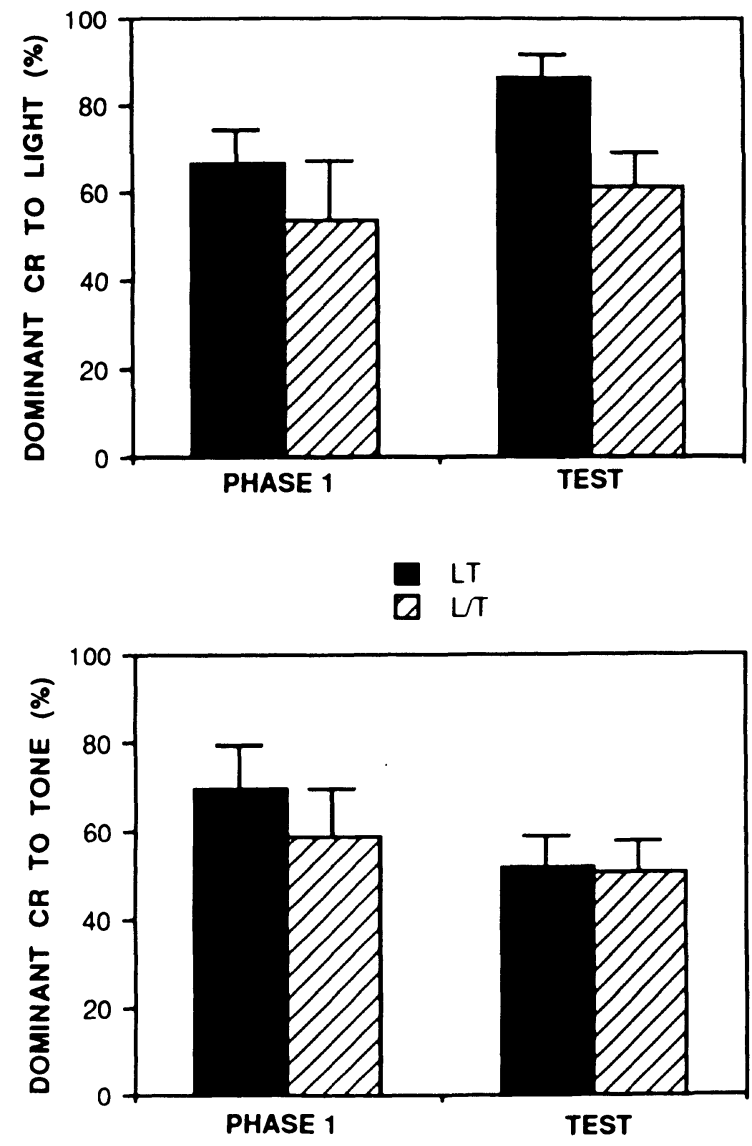

Figure 1. The probability of the occurrence of the dominant form of the CR to both the light and the tone before and after compound conditioning.

results of this comparison are shown in Figure 1. It was found that the dominant form of the CR to the light and the tone CSs were rearing and head-jerking, respectively, consistent with the findings of Holland (1977).

On the last day of individual conditioning (Phase 1) to the light and prior to compound conditioning, a MannWhitney $U$ test revealed that there was no significant difference between Groups LT and L/T $(U=32.0$, $p>.20, n=16$ ). Similarly, there was no significant difference between Groups LT and L/T in conditioning to the tone $(U=38.0, p>.20, n=16)$. An analysis of variance (ANOVA) revealed that there was no significant difference between groups LT and L/T for either stimulus $(F \mathrm{~s}<1)$.

It was found that after compound conditioning (Phase 2), Group LT showed a significant increase in the dominant form of the CR to the light [Wilcoxon $T(7)=$ $2, p<.05]$. These results were also confirmed by an ANOVA $[F(1,7)=8.00, p<.03]$. Using both nonparametric and parametric tests, no significant changes were observed for the dominant form of the CR to the light for Group L/T $[T(7)=15, p>.05 ; F(1,7)=.20]$. Group LT did not demonstrate a significant decrease in the dominant form of the CR to the tone $[T(7)=15$, $p>$.05]. However, an ANOVA did reveal a significant decrease in the dominant form of the CR to the tone $[F(1,7)=5.65, p<.05]$. The effect of compound conditioning on the tone is unclear given that different results occurred with parametric and nonparametric tests. Group L/T did not show a significant change in the dominant form of the $\mathrm{CR}$ to the tone $[T(8)=10, p>.05$; $F(1,7)=.44]$.

During the test, there was a significant difference between Groups LT and L/T with respect to the dominant form of the CR to the light $(U=10, p<.025, n=16)$. An ANOVA also confirmed this result $[F(1,14)=7.49$, $p<.02]$. There was no significant difference between Groups LT and L/T with respect to the dominant form of the CR to the tone $[U=29.50, p>.05, n=16 ; F(1,14)$ $=.91]$.

\section{DISCUSSION}

The results obtained with the light stands in contrast to the findings of Rescorla (1970) and the predictions of Rescorla and Wagner (1972). The present study found that when two CSs (which produced two dissimilar dominant CRs) were given excitatory conditioning as separate elements and then reinforced in compound, there was a tendency toward a decrement in the dominant form of the $\mathrm{CR}$ to the tone, whereas there was actually an increase in the dominant form of the CR to the light. Thus, the rules that govern stimulus compounding in a classical conditioning paradigm that employs two dissimilar CRs for the CSs may be different from those that govern the conditioned suppression procedure. In this way, the present findings may restrict the domain of the predictions made by the Rescorla-Wagner theory to CSs evoking CRs on a single response dimension or topography.

A possible explanation for these data could be proposed. The RescorlaWagner model may predict a loss of associative strength when the US is overexpected. In such situations, all the CSs present are simultaneously competing for the limited associative strength of the same US, giving rise to a CR on a unitary response dimension. That is, within a single CR dimension, such as conditioned suppression, one asymptote of conditioning must support conditioning among competing CSs. This suggests that the Rescorla-Wagner model could be viewed as a CRcompetition model in which CSs compete for associative strength based upon a unitary response dimension.

In the present study, two CSs with dissimilar CRs were utilized. Although both of these CSs were followed by the same magnitude of reinforcer, they were not necessarily in competition for a share of the limited associative strength, because the CSs evoked CRs on different response dimensions. Consequently, each CS should remain at asymptote (such as what may have occurred for the tone) or continue to gain associative strength (as occurred for the light) over the course of compound conditioning. It should also be noted that the increase in the CR to the light CS, as a result of compound training, may be due to the development of within-compound associations (e.g., Rescorla \& Durlach, 1980).

Additionally, it should be noted that the present findings were obtained by analyzing the specific form of the CR elicited by different CSs. This confirms the value of attending to the topographical features of the CR in order to expose processes of conditioning that are inexplicable in terms of traditional models of conditioning and are not manifest with the more traditional measures.

\section{REFERENCES}

Bindra, D., \& Blond, J. (1958). A time-sample method for measuring general activity and its components. Canadian Journal of Psychology, 12, 74-76.

Hilgard, E. R., MARQuis, D. G. (1940). Conditioning and learning. New York: Appleton-Century. 
Holland, P. C. (1977). Conditioned stimulus as a determinant of the form of the Pavlovian conditioned response. Journal of Experimental Psychology: Animal Behavior Processes, 3, 77-103.

Holland, P. C., \& Rescorla, R. A. (1975a). The effects of two ways of devaluing the unconditioned stimulus after first- and second-order appetitive conditioning. Journal of Experimental Psychology: Animal Behavior Processes, 1, 355-363.

Holland, P. C., Rescorla, R. A. (1975b). Second-order conditioning with food unconditioned stimulus. Journal of Comparative \& Physiological Psychology, 88, 459-467.

Jenkins, H. M., MoORE, B. R. (1973). The form of the auto-shaped response with food or water reinforcers. Journal of the Experimental Analysis of Behavior, 20, 165-181.

KAMIN, L. J. (1968). Attention-like processes in classical conditioning. In M. R. Jones (Ed.), Miami Symposium on the prediction of behavior: Aversive stimulation (pp. 9-31). Miami: University of Miami Press.

KAmin, L. J. (1969). Predictability, surprise, attention, and conditioning. In R. Church \& B. Campbell (Eds.), Punishment and aversive behavior (pp. 279-296). New York: Appleton-Century-Croft.

KREMER, E. F. (1978). The Rescorla-Wagner model: Losses in associative strength in compound conditioned stimuli. Joumal of Experimental Psychology: Animal Behavior Processes, 1, 22-36.

Rescorla, R. A. (1970). Reduction in the effectiveness of reinforcement after prior excitatory conditioning. Learning \& Motivation, 1 , 372-381.

Rescorla, R. A. (1989). Redundant treatments of neutral and excitatory stimuli in autoshaping. Journal of Experimental Psychology: Animal Behavior Processes, 3, 212-223.

Rescorla, R. A., \& Durlach, P. J. (1980). Potentiation rather than overshadowing in flavor-aversion learning: An analysis in terms of within-compound associations. Journal of Experimental Psychology: Animal Behavior Processes, 2, 175-187.

Rescorla, R. A., \&olomon, R. L. (1967). Two-process learning theory: Relationships between classical conditioning and instrumental learning. Psychological Review, 74, 151-181.

Rescorla, R. A., \& Wagner, A. R. (1972). A theory of Pavlovian conditioning: Variations in the effectiveness of reinforcement and nonreinforcement. In A. H. Black \& W. F. Prokasy (Eds.), Classical conditioning II: Current research and theory (pp. 65-99). New York: Appleton-Century-Croft.

Schachtman, T. R., Kasprow, W. J., Chee, M. A., \& Miller, R. R. (1985). Blocking but not conditioned inhibition results when an added stimulus is reinforced in compound with multiple pretrained stimuli. American Journal of Psychology, 98, 283-295.

SHETTLEWORTH, S. J. (1975). Reinforcement and the organization of behavior in the golden hamsters: Hunger, environment, and food reinforcement. Journal of Experimental Psychology: Animal Behavior Processes, 1, 56-87.

TerRY, W. S., \& WAGNER, A. R. (1975). Short-term memory for " surprising" versus "expected" unconditional stimuli in Pavlovian conditioning. Journal of Experimental Psychology: Animal Behavior Processes, 1, 122-133.
WAGNer, A. R., Rudy, J. W., \& WhitLow, J. W. (1973). Rehearsal in animal conditioning. Journal of Experimental Psychology, 97, 407-426.

\section{APPENDIX}

\begin{tabular}{|c|c|}
\hline Category Name & Description \\
\hline Preambulate & $\begin{array}{l}\text { Changes in position involving all four } \\
\text { feet, including walking across chamber, } \\
\text { circling, and/or jumping suddenly to } \\
\text { another position; often accompanied by } \\
\text { sniffing. }\end{array}$ \\
\hline
\end{tabular}

Rear

Standing on hind legs with both forepaws off the grid floor, usually (not always) stretching to full extent, forepaws usually (not always) on top of side walls; may be accompanied by sniffing or slow side-toside movement of head. Does not include grooming.

Magazine Standing motionless in front of food magazine with nose or head within magazine, sometimes (rarely) gnawing on edges of magazine opening.

Head-jerk Short rapid horizontal and/or vertical movements of the head, usually oriented toward food magazine; hindquarters motionless. Infrequently occurring with rear. In those cases only head-jerk scored.

Head-jerk/hind Head-jerk plus movement of hindquarters, either side-to-side or foward-backward. Simultaneous display of head-jerk and perambulate (rare) also scored as headjerk/hind.

Other Grooming head, body, or tail; scratching; gnawing grid bars; standing motionless with head above or between grid bars; lying with abdomen on grid floor; sniffing (provided rat not also performing one of above behaviors).

(Manuscript received March 19, 1990.) 